\title{
Cultural Citizenship
}

Ein kommunikationswissenschaftliches Konzept zur Bestimmung kultureller Teilhabe in der Mediengesellschaft

\section{Elisabeth Klaus / Margreth Lünenborg}

Der Beitrag führt das Konzept „cultural citizenship“ als konstitutive Dimension von "citizenship“ in der Mediengesellschaft ein. Anknüpfend an Thomas M. Marshalls dreidimensionale Unterscheidung von "Staatsbürgerschaft" als „civil, political and social citizenship“ werden mit dem Begriff der „cultural citizenship“ jene Praxen bezeichnet, die eine Teilhabe an den kulturellen Ressourcen der Gesellschaft ermöglichen. Der Begriff bietet damit die Möglichkeit, mediale Entwicklungen und damit zusammenhängende gesellschaftliche Phänomene zugleich auf der Mikro- wie auf der Makroebene zu erfassen. Am Beispiel von zwei unterschiedlichen Anwendungsfeldern (Reality TV und Weltinformations-Gipfel-WSIS) wird die Aussagefähigkeit des theoretischen Konzeptes und seine Relevanz für die Kommunikationswissenschaft erörtert.

Keywords: Cultural Citizenship, Mediengesellschaft, Identitäten, Reality TV, World Summit Information Society, Staatsbürgerkonzept, Medientheorie

\section{Einleitung}

Die Zugehörigkeit zu einer Gemeinschaft wird heute zentral über Medien verhandelt. $\mathrm{Ob}$ als Mitglied einer Clique oder einer Fangruppe, ob als Kriegsgegnerin oder als Aktienbesitzer, als Mitglied eines Nationalstaates oder der Weltgesellschaft: Medien und spezifische Formen ihrer Nutzung sind unverzichtbare Bestandteile zur Konstituierung von Identität(en). Beschreibt man die aktuelle Formation der Gesellschaft als Mediengesellschaft, so werden sowohl nationale Zugehörigkeit wie auch Partizipationschancen in der Weltgesellschaft maßgeblich über Teilhabe an Formen der medialen Kommunikation bestimmt. Diese zentrale Rolle der Medien bei der Verhandlung symbolischer Ressourcen der Gesellschaft drückt sich aus in einer Veralltäglichung der Medieninhalte und alltagsgebundenen Formen der Rezeption. Gleichzeitig werden zentrale politische und wirtschaftliche Diskurse zunehmend medial konstituiert und inszeniert.

Wir entwickeln nachfolgend in Auseinandersetzung mit vorliegenden englischsprachigen Texten das Konzept einer cultural citizenship und versuchen, dieses erstmals kommunikationswissenschaftlich zu fundieren. Dieses Konzept ermöglicht es, die Bedeutung von Medien bei der Konstituierung individueller wie gesellschaftlicher Identität gleichzeitig auf einer Mikro- wie auf einer Makroebene zu analysieren. Cultural citizenship liefert Möglichkeiten, einerseits individuelle Prozesse der Medienrezeption zu betrachten, andererseits Makroprozesse der Konstituierung nationaler und globaler Medienöffentlichkeit. Der große Vorteil davon ist, dass damit in der Kommunikationswissenschaft unter Bezug auf theoretische Konzepte der Cultural Studies Diskurse zur Integrationsleistung von Medien zusammengeführt werden, die bislang scheinbar unvereinbar nebeneinander existierten. Die Relevanz, die Aussagekraft und die Konsequenzen des Konzeptes cultural citizenship werden wir exemplarisch an zwei entsprechend heterogenen Anwendungsfeldern diskutieren: an aktuellen Genres des Fernsehjournalismus (Reality TV) einerseits sowie aktuellen politischen Debatten, wie sie 
anlässlich der UN-Weltkonferenz zur Informationsgesellschaft (WSIS - World Summit on the Information Society) stattgefunden haben bzw. weiterhin stattfinden, andererseits.

\section{Dimensionen von Citizenship}

Der deutsche Begriff der Staatsbürgerschaft rückt die politischen Rechte der Bürgerinnen und Bürger gegenüber dem Staat in den Mittelpunkt. Politische Beteiligungsrechte erscheinen damit als Dreh- und Angelpunkt der Zugehörigkeit von Individuen zum Nationalstaat und gelten als primäre Bedingung einer funktionierenden Demokratie. Demgegenüber ist der englische Begriff citizenship deutlich weiter angelegt. Er fokussiert besonders die zivilen Rechte der Bürgerinnen und Bürger und macht damit das Spannungsverhältnis zwischen Staat und Zivilgesellschaft sichtbar.

Der einflussreiche englische Soziologe Thomas H. Marshall stellte bereits 1949 ein differenziertes Modell von citizenship vor. Er skizziert drei Dimensionen von citizenship und unterscheidet entsprechend civil, political und social citizenship (Marshall 1992: 39 ff.). Obwohl er mit dieser Differenzierung die soziologische und politikwissenschaftliche Diskussion um „Staatsbürgerschaft" im englischsprachigen Raum maßgeblich bestimmt hat, wurde er in der deutschen Debatte bis heute kaum rezipiert (vgl. Rieger 1992: 22). ${ }^{1}$ Nachfolgend soll deshalb Marshalls Konzept von citizenship dargestellt und knapp diskutiert werden.

Als civil citizenship beschreibt Marshall (1992: 40) jene „Rechte, die notwendig sind, die individuelle Freiheit zu sichern". Konkret umfasst sie damit die Freiheit der Person, Rede-, Gedanken-, Glaubens- und Pressefreiheit, die Freiheit des Eigentums sowie das Recht auf ein Gerichtsverfahren. Der Bürger im Staat wird damit als homo oeconomicus gesetzt. Seine autonome wirtschaftliche Handlungsfähigkeit ist Voraussetzung für die Weiterentwicklung der Gesellschaft. Der freie Bürger ist also von Anfang an gleichzeitig citizen und consumer. Gerichte werden als Instanz zur Durchsetzung der bürgerlichen Rechte etabliert. Political citizenship benennt das Recht auf Teilhabe am Gebrauch der politischen Macht und drückt sich im aktiven und passiven Wahlrecht aus. Das Parlament wird damit zur wesentlichen Instanz der Realisierung von politischen Rechten. Social citizenship schließlich umfasst das Recht auf ein Mindestmaß an wirtschaftlicher Wohlfahrt und Sicherheit sowie auf einen Anteil am gesellschaftlichen Reichtum. Der Wohlfahrtsstaat mit seinem Erziehungswesen und den sozialen Diensten ist für Marshall die Instanz, mittels derer sich soziale Rechte realisieren.

Marshall beschreibt die historische Entstehung der drei Dimensionen von citizenship als chronologische Entwicklung, die sich in einem „doppelte[n] Prozeß der Verschmelzung und Trennung" (1992: 41) ausbildet. Während geographisch ein Prozess der Verschmelzung einsetzt, um nationale Rechte ausgehend von lokalen Strukturen in Dorfgemeinschaften, Städten oder Zünften zu etablieren, findet gleichzeitig eine funktionale Trennung statt. So weist Marshall (1992: 51) nach, dass die sozialen Rechte von zivilen

1 Ralf Dahrendorf (1957), dessen Dissertation von Marshall betreut wurde, nutzte als einer der ersten dessen Überlegungen für eine Strukturanalyse der modernen Gesellschaft (vgl. Rieger 1992: 22). Dennoch ist die Auseinandersetzung mit den differierenden citizenship-Konzepten damit in Deutschland nicht vorangetrieben worden. Dominant bleibt bis heute die Konzentration auf politische Beteiligungsrechte. Nicht zufällig liegt deshalb eine deutsche Übersetzung von Marshalls 1950 veröffentlichtem Vortrag erst seit 1992 vor. 
und politischen Rechten abgelöst wurden, um erst im 20. Jahrhundert als soziales Sicherungssystem in neuer Form wieder zu entstehen. ${ }^{2}$ Die Etablierung ziviler Rechte verortet Marshall im 18. Jahrhundert. In dieser Zeit entwickelte sich die Freiheit des Individuums gegenüber dem Staat. „In den Städten waren die Begriffe ,Freiheit' und „Bürgerrecht' austauschbar. Als die Freiheit allgemein wurde, entwickelte sich das Bürgerrecht von einer lokalen zu einer nationalen Institution“"(Marshall 1992: 45f.). Das 19. Jahrhundert beschreibt Marshall als Zeitraum der Etablierung politischer Rechte. Dabei ging es vor allem um die Ausweitung alter Rechte auf neue Bevölkerungsgruppen. Demnach war 1918 das „allgemeine Männerwahlrecht“ (Marshall 1992: 47) durchgesetzt, da von nun an nicht länger Herkunft und Besitz, sondern allein die bürgerliche Existenz die Teilhabe an politischen Entscheidungen ermöglichte.

Obwohl Frauen ebenfalls 1918 das Wahlrecht erhielten, verfügten sie zu diesem Zeitpunkt weder über vollständige bürgerliche noch politische Rechte. Diese Tatsache weist bereits darauf hin, dass Marshalls Deutung einer sich historisch kontinuierlich ausweitenden citizenship nicht für alle Bevölkerungsgruppen zutreffend ist. Im 20. Jahrhundert schließlich wurden soziale Rechte als nationale staatliche Leistungen verankert, nachdem sie im Mittelalter partiell in lokalen Gemeinschaften und Zweckverbünden anerkannt waren. Neben dem sozialen Fürsorgesystem erscheint Marshall hier vor allem das Bildungswesen zentral. Der gleiche Zugang zu Bildungsressourcen ermöglicht den $\mathrm{Zu}-$ tritt zur bürgerlichen Gesellschaft. „Bildung ist eine unverzichtbare Voraussetzung der bürgerlichen Freiheit“ (Marshall 1992: 51). Aus dem Recht auf Bildung erwächst das staatliche Interesse an Bildung seiner Staatsbürger, der Staat erlässt die Schulpflicht. „Die Pflicht zum eigenen Fortschritt und zur eigenen Zivilisierung ist eine soziale Pflicht, und nicht nur eine persönliche, weil die soziale Gesundheit der Gesellschaft von der Kultur ihrer Mitglieder abhängig ist. Und eine Gesellschaft, die diese Pflicht durchsetzt, beginnt zu erkennen, daß ihre Kultur eine organische Einheit und ihre Zivilisation ein nationales Erbe ist" (ebd.). An dieser Stelle bietet Marshall bereits eine Verbindung zu einer vierten Dimension von citizenship, der cultural citizenship an, die uns nachfolgend beschäftigen wird. Marshall selbst führt diesen Begriff jedoch nicht ein.

Fraser und Gordon (1994) stellen in einer kritischen Diskussion zum Verhältnis von civil und social citizenship heraus, dass Marshalls dreidimensionales Modell nicht allein eine Beschreibung der historischen Entwicklung von citizenship darstellt, sondern zugleich eine soziale Utopie enthält. Marshall beschreibt mit der Entwicklung von bürgerlichen, politischen und sozialen Rechten die Vision einer zunehmend gerechteren und solidarischeren Gemeinschaft. Eine solche gradlinige Entwicklung stellen Fraser und Gordon grundlegend in Frage: „When questions about gender and race are put at the center of the enquiry, key elements of Marshall's analysis become problematic. His periodization of the three stages of citizenship, for example, fits the experience of white working men only, a minority of the population“ (Fraser/Gordon 1994: 93). So war in den USA die Erlangung der Bürgerrechte von weißen Männern mit einem Verlust an Persönlichkeitsrechten für (verheiratete) Frauen und für die schwarze Bevölkerung

2 So verloren Bewohner des Armenhauses in England bis 1918 ihre zivilen und persönlichen Rechte. „Das Armenrecht behandelte die Anrechte der Armen nicht als integrativen Bestandteil der Rechte eines Bürgers, sondern als Alternative zu ihnen - als Ansprüche, die nur dann befriedigt werden konnten, wenn der Anwärter aufhörte, ein Bürger in jedem wahren Sinne des Wortes zu sein" (Marshall 1992: 49f.). Dagegen hatte das Armenrecht der Elisabethanischen Gesetzgebung bereits konzeptionell dem Modell eines modernen Wohlfahrtstaats entsprochen. 
durch Sklaverei verbunden. Deren rechtliche und ökonomische Abhängigkeit wurde zur Voraussetzung für die Entwicklung bürgerlicher Freiheitsrechte. Während eine solche civil citizenship auf der Idee eines Vertrages zwischen Gleichberechtigten basiert, gründen die sozialen Rechte auf der Norm einer Fürsorge für Schwächere. „[T] gemony of contract helped to generate a specifically modern conception of ,charity' as its complementary other" (Fraser/Gordon 1994: 101).

Blickt man also auf die verschiedenen gesellschaftlichen Gruppen, so lässt sich Marshalls Modell der linearen und additiven Entwicklung bürgerlicher, politischer und sozialer Rechte nicht aufrecht erhalten. Vielmehr handelt es sich um einen widersprüchlichen und ungleichzeitigen Prozess, in dem beispielsweise Frauen und ethnische Minderheiten verspätet und bis heute unvollständig an citizenship teilhaben. Das dreidimensionale Modell macht jedoch sichtbar - das ist sein großes Verdienst -, dass die Zugehörigkeit zur nationalstaatlichen Gemeinschaft auf weitaus mehr gründet als allein auf politischen Rechten und Pflichten. Citizenship erschöpft sich keinesfalls in „Staatsbürgerschaft", sondern bedeutet Zugehörigkeit zu wirtschaftlich, sozial, kulturell und räumlich verorteten Gemeinschaften.

\section{Zugehörigkeit in der Mediengesellschaft mittels Cultural Citizenship}

Verschiedene AutorInnen, überwiegend aus dem englischsprachigen Raum, haben versucht, Marshalls Typologie für die moderne und postmoderne Gesellschaft fortzuschreiben (vgl. für eine Rezeptionsgeschichte Marshall 1992: 189-195). Seit etwa zehn Jahren taucht dabei gelegentlich der Begriff der cultural citizenship ergänzend zu den bisherigen drei Dimensionen auf. Er scheint uns geeignet, die Bedeutung der Massenmedien für die aktuelle Konstituierung der citizens zu erfassen, auch wenn nur einige der im Folgenden vorgestellten AutorInnen diese Verbindung selber herstellen. Die verstärkte Berücksichtigung der kulturellen Dimension des Sozialen in den 90er Jahren des 20. Jahrhunderts trägt vermutlich zu einem vermehrten Gebrauch des Begriffes der cultural citizenship bei, ohne dass das Konzept deshalb einheitlich verstanden würde. Nur wenige AutorInnen beziehen sich in ihren Ausarbeitungen aufeinander, und auch der Ursprung des Begriffes lässt sich nicht eindeutig bestimmen. ${ }^{3}$ Bei allen Differenzen und Ungenauigkeiten verweist der gemeinsam verwendete Begriff cultural citizenship jedoch auf einen konsensuellen Kern, der Möglichkeiten eröffnet, die zentrale Bedeutung der Medien für die heutige Konstituierung von (Staats-)Bürgerschaft zu erfassen.

In seinem Beitrag „Postmodern Culture/Modern Citizens“ erweitert Bryan S. Turner 1994 Marshalls Typologie um den Begriff der cultural citizenship. Dabei definiert Turner citizenship ,as a set of practices which constitute individuals as competent members of society" (Turner 1994: 159). Turner hebt mit dieser soziologischen Definition hervor, dass StaatsbürgerInnen als Mitglieder einer Gemeinschaft durch die verschiedenen sozialen, rechtlichen, politischen und kulturellen Praxen konstituiert werden bzw. sich selber konstituieren. Er grenzt sich damit ab von der insbesondere auch in Deutsch-

3 Manchmal wird der Begriff prominent verwendet, ohne dass er deshalb näher erläutert würde. So nennt etwa Toby Miller (1998) sein Buch „Technologies of Truth. Cultural Citizenship and the Popular Media“, eine genaue Definition des Begriffes oder eine pointierte Diskussion von cultural citizenship sucht man darin aber vergeblich. Seine Ausführungen über Nationalstaat, Publikum, Genre und populäre Medien in Moderne und Postmoderne lassen allenfalls erahnen, worauf der Titel zielt. 
land verbreiteten Betonung der mit Staatsbürgerschaft verbundenen juristischen und politischen Rechte, die an geeignete AspirantInnen verliehen werden. Demgegenüber besitzt citizenship Turners Definition zufolge sowohl eine aktive wie passive Seite, weil Identität als citizen in der Verbindung aus Selbstbeschreibung und Fremdzuschreibung entsteht. Turner definiert: „Cultural citizenship consists of those social practices which enable a competent citizen to participate fully in the national culture" (ebd.). Unter Kultur verstehen wir dabei den je historisch und sozial gebundenen Vorrat an symbolischen Deutungs- und Interpretationsmöglichkeiten, der innerhalb eines Nationalstaats bzw. der Weltgesellschaft zur Verfügung steht (vgl. Thompson 1990). Für Grossberg (1999: 23) übernimmt Kultur damit die Funktion einer „Vermittlungsinstanz, durch welche das Chaos der Realität in den geordneten Sinn der menschlichen Realität verwandelt wird“. Gleiche Bildungschancen, die Marshall als Bestandteil der social citizenship gefasst hat, erklärt Turner zum zentralen Element von cultural citizenship. Das ist insofern theoriekonsistent, weil Bildung - in der umfassenden Bedeutung des „lebenslangen Lernens“weniger einen wohlfahrtsstaatlichen Anspruch formuliert als eine bedeutende symbolische Ressource darstellt, ohne die citizenship nicht zu verwirklichen ist.

Auch wenn Turner die Massenmedien in seinem Aufsatz nicht erwähnt, so leuchtet doch unmittelbar ein, dass der Zugang zu den Massenmedien heute eine bedeutende, wenn nicht die zentrale kulturelle Ressource darstellt. ${ }^{4}$ Auch der britische Medienwissenschaftler John Hartley argumentiert entsprechend: „Television is used, both in its original ,mass' broadcast form, and now in its emergent subscriber-choice forms, to teach two new forms of citizenship, which I am calling, cultural' and,do-it-yourself' or DIY citizenship respectively." (Hartley 1999: 154f.; Hervorh. i. O.) Hartley folgend gehörte cultural citizenship zu einer nahezu abgeschlossenen Phase der Mediengesellschaft, in der das Fernsehen als gesellschaftliches Massen- und Leitmedium die Formierung einheitlicher Identitäten gefördert hat. Das heutige Fernsehen versammelt dagegen nicht mehr die Nation vor dem Bildschirm, sondern mit der Vielfalt an Sparten- und Zielgruppenangeboten zerfällt das kollektive Fernsehpublikum in Individuen und Interessengruppen, die sich ihre jeweils eigenen Medienmenüs zusammenstellen. Die Postulierung der „DIY citizenship“ als einer neuen Phase in der Entwicklung von citizenship erscheint uns jedoch als voreilig, ist doch das Fernsehen immer noch auch Initiator gemeinsamer nationaler Medienereignisse. Gerade in Zeiten der Fragmentierung sind Massenmedien für eine konsensuale gesellschaftliche Bedeutungsproduktion und Sinnstiftung konstitutiv. Sie dienen wesentlich der Bereitstellung nationaler wie auch Nationen übergreifender symbolischer Ressourcen und sozial gebundene Deutungsmuster.

Festzuhalten ist, dass Hartley die Entwicklung von citizenship in der Moderne als grundlegend medial vermittelt charakterisiert: „(C)itizenship is profoundly mediated in the modern/postmodern period - we are all , citizens of the media' (Hartley 1996: Kap. $\mathrm{XX}$ ) in the sense that participation in public decision-making is primarily conducted through media“ (Hartley 1999: 157). Im Unterschied zum traditionellen Nationalstaat ist in der Mediengesellschaft nicht der mit politischen Rechten und Pflichten ausgestattete Staatsbürger der Souverän, sondern das sozial situierte und kulturell kontextuierte Publikum. Ursachen und Folgen dieser Veränderung werden beispielsweise in der Po-

4 Hier ergibt sich eine unmittelbare Verbindung zu den britischen Cultural Studies, für die Bildung/Erziehung und Massenmedien die beiden zentralen Instanzen sind, mittels derer der symbolische Kampf um Bedeutung ausgefochten wird (beispielhaft Morley 1999, 2002; Storey 2003). 
litikwissenschaft im Kontext „symbolischer Politik“ (Sarcinelli 1987) bzw. mediatisierter Politik (vgl. Dörner 2000, 2001 und Meyer u. a. 2000) ausgiebig diskutiert. Das „Citizenship“-Modell erweitert sich vor allem via Medien um die Dimension der cultural citizenship. Für das als Konsument wie als Staatsbürgerin angesprochene Publikum der Massenmedien wird cultural citizenship neben der bürgerlichen, politischen und sozialen citizenship zentrale Voraussetzung für gesellschaftliche Zugehörigkeit.

Hartley und Turner positionieren beide cultural citizenship auf der Seite der Moderne. Für Hartley reicht das Konzept dabei im Unterschied zu Turner über das Gleichheitspostulat, das gleiche Rechte für alle BürgerInnen fordert, hinaus. Differenz sei vielmehr ebenso als Menschenrecht anzuerkennen wie das Recht auf Gleichheit (vgl. Hartley 1999: 164). Vor allem feministische und antirassistische Theorien haben hervorgehoben, dass Gleichberechtigung, wenn sie mehr als ein formaler Anspruch sein soll, zu ihrer Verwirklichung die Anerkennung von Differenz, von unterschiedlichen Ausgangsbedingungen und Entfaltungsmöglichkeiten der Individuen voraussetzt. Hier setzt Renato Rosaldo (1994) mit seinen Überlegungen an: Für ihn besteht gerade in der Anerkennung von Differenz die Essenz des Konzeptes von cultural citizenship. Entsprechend definiert er es als "the right to be different (in terms of race, ethnicity, or native language) without compromising one's right to belong, in the sense of participating in the nation-state's democratic processes“ (Rosaldo 1994: 57). Als vordergründig universelles Prinzip setzt Staatsbürgerschaft ein weißes, männliches, heterosexuelles Subjekt voraus und negiert die Marginalisierung und den Ausschluss anderer Gruppen (vgl. Fraser/Gordon 1994). Nach Rosaldo bedeutet cultural citizenship die Anerkennung jener kulturell vermittelten Annahmen und Praxen, die Ungleichheit gerade auf der Basis rhetorischer Gleichheit begründen und verfestigen. Cultural citizenship liefert damit auch Ausdrucksmöglichkeiten für die Belange marginalisierter Gruppen, die nicht karitative Unterstützung, sondern gesellschaftliche Anerkennung einfordern: „Bridging the discourses of the state and everyday life, of citizenship and culture, the demand for respecto is a defining demand of cultural citizenship." (Rosaldo 1999: 260)

Auch wenn Rosaldo sich nicht mit Massenmedien beschäftigt, so ist zumindest unter einer Cultural Studies-Perspektive klar, dass Medien dafür die Voraussetzungen bereitstellen. Sie sind einerseits Vermittler zwischen öffentlichem und privatem Leben, andererseits stellen sie die Mittel bereit für das identitätsstiftende Spiel zwischen kultureller Vereinheitlichung und subkultureller Besonderung. Graham Murdock (1999: 10) sieht das Fernsehen entsprechend als „the principal stock exchange of public discourse“. Dort werden Ansprüche auf Anerkennung der Differenz ausgehend von einem gemeinsam konstituierten öffentlichen Raum formuliert. Joke Hermes (1998: 159), die sich dabei auf Turners Ausführungen zu cultural citizenship bezieht, hat in diesem Zusammenhang auf das bedeutende politische Potenzial insbesondere populärer und fiktionaler Genres für gesellschaftlich nicht-dominante Gruppen verwiesen.

Dabei scheint sie jedoch wie Rosaldo die Machtdimension bei der Formierung von cultural citizenship und ihre Entstehung im Zusammenwirken von Selbst- und Fremdzuschreibung zu negieren. Macht bedeutet für uns in diesem Kontext ein Gesellschaftsverhältnis, das ungerechte Ressourcenverteilung und eine hierarchische Beziehung zwischen verschiedenen Gesellschaftsgruppen beinhaltet. Machtverhältnisse werden nichtdominanten Gruppen aber nicht lediglich von außen oktroyiert. Ihre bindende Wirkung erhalten sie vielmehr durch das weit gehende Einverständnis dieser Gruppen mit hegemonialen Rechtfertigungsmustern. Dieses Problem hat die Ethnologin Aihwa Ong (1999a, 1999b, 1996) ausgearbeitet. Sie wählt einen ähnlichen Ausgangspunkt wie Rosaldo, demzufolge differente Positionierungen mittels kultureller Prozesse ausgedrückt 
werden. Ong beschäftigt sich mit der Frage, wie citizenship unter den Bedingungen von Globalisierung und Transnationalität entsteht. Sie entwirft einen analytischen Rahmen, der es ermöglicht, die ökonomischen Globalisierungsprozesse zu erfassen und zugleich die kulturelle Dynamik der politischen Reaktionen darauf zu beschreiben (vgl. Ong 1999a). Vor diesem Hintergrund versteht sie unter cultural citizenship „the cultural practices and beliefs produced of our negotiating the often ambivalent and contested relations with the state and its hegemonic forms that establish the criteria of belonging within a national population and territory. Cultural citizenship is a dual process of selfmaking and being-made within webs of power linked to the nation-state and civil

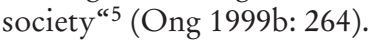

Mit dieser dialektischen Bestimmung von cultural citizenship als aktivem und passivem, als selbst- und fremdbestimmtem Prozess grenzt Ong sich bewusst von Rosaldo ab. Sein Konzept, so kritisiert sie, schüre die Illusion „that immigrant or minority groups can escape the cultural inscription of state power and other forms of regulation that define the different modalities of belonging" (Ong 1999b: 264). Den Machtaspekt bei der Konstituierung gesellschaftlicher Identität hebt gleichermaßen Terkessidis (1999) in Auseinandersetzung mit dem Begriff der „Hybridkultur“ hervor. Er kritisiert jene Konzepte, die die „Vermischung“ von Kulturen naiv als gleichwertiges Nebeneinander darstellen: „Offenkundig dient die harmonistische Verwendung des Hybriditätsbegriffs in erster Linie dazu, die neue Identitätskonstruktion der Mehrheit zu erfassen.“ (Terkessidis 1999: 239) Auch wenn die jeweilige nationalstaatliche Kultur einer Vielzahl von Einflüssen unterliege, so setze dies die fortbestehende Hierarchie kultureller Deutungsmacht nicht außer Kraft.

In den vorliegenden Ausführungen zu cultural citizenship zeigen sich bei aller Differenzierung zwei wichtige Übereinstimmungen: Erstens wird die Bedeutung der kulturellen Praxen, der jeweiligen Interpretations- und Deutungsmuster, für die Teilhabe an der Gesellschaft betont und zweitens werden die damit aufgeworfenen Fragen des $\mathrm{Zu}-$ gangs zu den symbolischen Ressourcen der Gesellschaft unter Bedingungen struktureller Ungleichheiten fokussiert. Diese analytische Inklusion des Machtbegriffs ist ein zentraler Bestandteil des Konzeptes cultural citizenship. Theoretische Bezugspunkte der Diskussion bilden die Cultural Studies und ihre Vorstellung von Dominanz und Hegemonie sowie Foucaults Begriffe von Macht und Diskurs. ${ }^{6}$ Ein tragfähiges Konzept von cultural citizenship muss dann neben der von Turner betonten Prozesshaftigkeit des Staatsbürgerwerdens und -seins weitere Erkenntnisse berücksichtigen: Das mit dem Staatsbürgerkonzept verbundene Gleichheitspostulat kann nur in der Anerkennung von Differenz wirksam werden. Differenz dient der hierarchischen Gliederung der Gesellschaft und ist ein Ausdruck gesellschaftlicher Machtverhältnisse. Differenz wird dabei in Identitätsbildungsprozessen mit hergestellt, die immer gleichzeitig fremd- und selbstbestimmt ablaufen. Weiter ist cultural citizenship in der Mediengesellschaft grundlegend medieninduziert und medienvermittelt. Auch wenn andere kulturelle Ressourcen der Gesellschaft zu ihrer Herausbildung beitragen, so ist in der gegenwärtigen (Medien-) Gesellschaft keine kulturelle Identität jenseits oder außerhalb der medial vermittelten Wirklichkeit möglich. Diese zentrale - aber nicht exklusive - Bedeutung von Medien

5 Der Begriff der „civil society“, auf den wir später noch eingehen werden, wird hier neben dem Nationalstaat als wichtiger Agens der Identitätskonstruktion eingeführt.

6 Wir erwähnen diese wissenschaftstheoretischen Bezugsgrößen des Begriffes der Vollständigkeit halber, wollen diese aber hier aus Platzgründen nicht genauer nachzeichnen. 
macht das Konzept cultural citizenship für die Kommunikationswissenschaft in besonderer Weise interessant. Daraus leiten wir eine Definition von cultural citizenship ab, die die kommunikationswissenschaftlichen Aspekte des Konzeptes besonders hervorhebt.

Cultural citizenship ist eine wesentliche Dimension von „Staatsbürgerschaft“ in der Mediengesellschaft. Sie umfasst all jene kulturellen Praktiken, die sich vor dem Hintergrund ungleicher Machtverhältnisse entfalten und die kompetente Teilhabe an den symbolischen Ressourcen der Gesellschaft ermöglichen. Massenmedien sind dabei Motor und Akteur der selbst- und zugleich fremdbestimmten Herstellung von individuellen, gruppenspezifischen und gesellschaftlichen Identitäten.

\section{Cultural Citizenship in der medienvermittelten Bedeutungsproduktion}

Cultural citizenship stellt demnach den unverzichtbaren Kontext dar, innerhalb dessen Medien in der und für die Gesellschaft relevant werden. Klaus und Lünenborg (2000) haben den Medienprozess als Kreislauf kultureller Bedeutungsproduktion gekennzeichnet. Anknüpfend an Johnson (1985) beschreiben sie damit, dass faktische wie fiktionale Medientexte gesellschaftliche Bedeutung im Zusammenwirken von Produktion, Medientext und Rezeption erhalten. Nicht der isolierte Medientext materialisiert gesellschaftliche Machtverhältnisse, vielmehr werden diese von den ProduzentInnen in den Text eingeschrieben und vom Publikum im Prozess der Rezeption dem Text zugewiesen. Dass dabei Differenzen zwischen den intendierten Medienaussagen der KommunikatorInnen, den hegemonialen Bedeutungen von Texten und den Lesweisen und Aneignungsleistungen des Publikums auftreten (können), ist im Rahmen der Cultural Studies ausgiebig diskutiert und auch empirisch gezeigt worden (vgl. für einen Überblick Ang 1998; Hepp/Winter 1999; Moores 1993; Morley 1980; Fiske 2001). Das Konzept cultural citizenship gewinnt in diesem Kreislauf an Bedeutung, weil es den Kontext liefert, in den sämtliche Elemente des Kreislaufs medialer Bedeutungsproduktion eingebunden sind. Cultural Citizenship spannt jenen Raum auf, in dem Bedeutungen „zirkulieren“, also verhandelt und festgelegt werden (vgl. Abb. 1).

Diese Beschreibung erscheint uns präziser als jene Konzeptionierung, die in der Studie von du Gay/ Hall et al. (1997) unter der Bezeichnung circuit of culture firmiert. Dort nehmen die Autoren Repräsentation, Identität und Regulation neben der Produktion und Rezeption von Medientexten in den Kreislauf auf. Unklar bleibt in dieser Struktur jedoch, welcher analytische Stellenwert ideologischen und sozialen Prozessen der Repräsentation und Identitätsbildung zukommt und welche Bedeutung ökonomische Prozesse der Regulation in und neben der Produktion und Rezeption haben. Wir plädieren deshalb dafür, Prozesse der individuellen, subkulturellen oder nationalstaatlichen Identitätsbildung als Kontext jeglichen Medienhandelns zu begreifen. Sie bilden den Hintergrund von Produktion wie Rezeption und finden sich als symbolische Bedeutung im Medientext eingeschrieben.

Cultural citizenship bestimmt wesentlich den Prozess gesellschaftlicher Identitätsbildung mit seinen Ein- und Ausschlüssen. Dieser ist medieninduziert und medienvermittelt. Nur wenn für die verschiedenen sozialen Gruppen mediale Angebote existieren, die eine diskursive Auseinandersetzung mit den jeweils vorhandenen kulturellen Praktiken ermöglichen und damit auch ihre Entwicklung und Modifizierung erlauben, kann gesellschaftliche Zugehörigkeit entstehen. Dabei sind mediale Diskursräume wesentlich an die jeweiligen Sprachräume gebunden. Möglichkeiten der verstehenden Partizipation 


\section{Abbildung 1: Cultural Citizenship als Kontext im Kreislauf kultureller Bedeutungsproduktion}

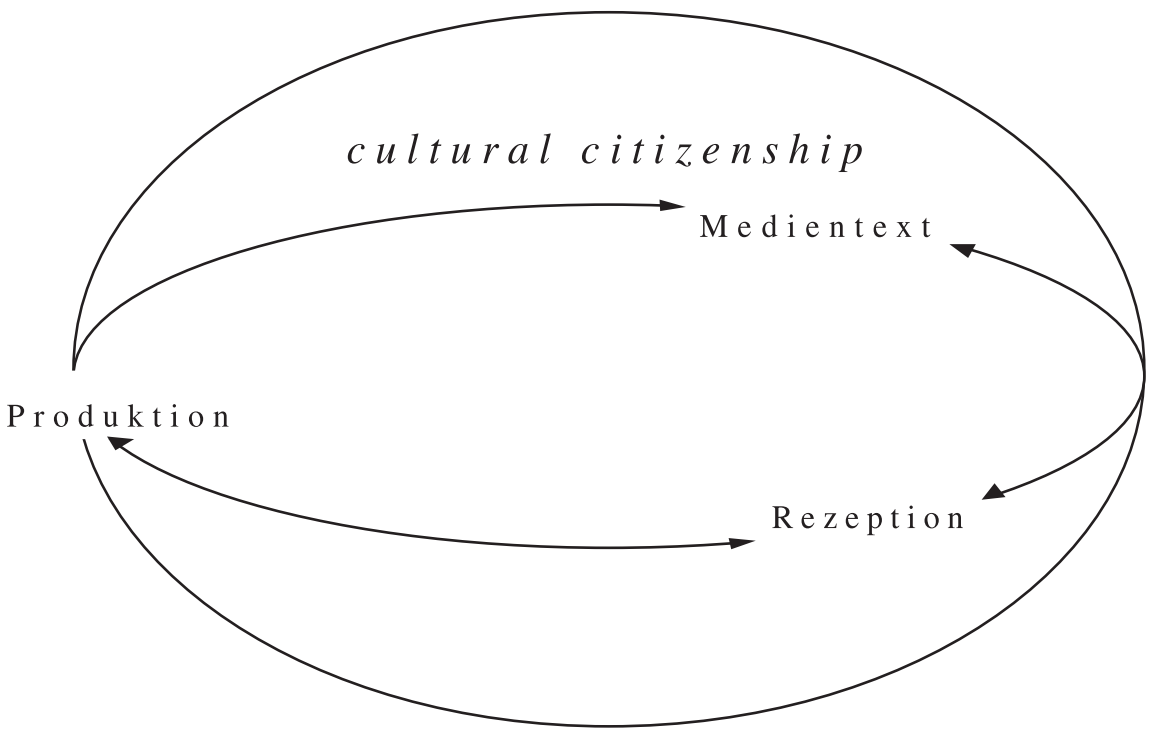

an den kulturellen Ressourcen des sozialen Umfeldes beruhen auf sprachlicher Verständigung. Ein solchermaßen begründeter Prozess kultureller Zugehörigkeit ist Voraussetzung für die umfassende Wahrnehmung von politischen oder sozialen Bürgerrechten.

Ein Beispiel soll das erläutern: Wo türkische Migrantinnen und Migranten keine Anknüpfungspunkte an die medialen Diskurse der deutschen Gesellschaft finden, wo ihren kulturellen Alltagspraktiken kein Raum im Mediendiskurs eingeräumt wird, da müssen sie sich entweder assimilieren und ihre eigene kulturelle Identität aufgeben oder es bleiben ihnen kulturelle Rechte als citizens verwehrt. Die Nutzung ausschließlich muttersprachlicher Medienangebote lässt die „kulturelle Staatenlosigkeit“ sichtbar werden und behindert auch die politische, soziale und zivile Teilhabe, selbst dann, wenn mit der deutschen Staatsangehörigkeit politische Partizipationsrechte erworben wurden. Somit tragen bspw. MTV-Moderatorinnen türkischer Herkunft, die subkulturelle Artikulationsformen massenmedial kommunizieren, unter Umständen stärker zur Herstellung von cultural citizenship bei als andere, traditionell höher bewertete Medientexte. Zu vermuten ist, dass es den kulturellen Diskursen der Musikindustrie, wie sie etwa die Medienangebote von MTV präsentieren, stärker als klassischen Informationsmedien gelingt sprachraumgebundene Grenzen nationaler Kulturen zu überschreiten. Die Populärkultur bietet auf diese Weise Anknüpfungspunkte für eine Differenz, die das Recht auf Teilhabe nicht verwehrt (vgl. dazu Hermes 1998; Klaus/O’Connor 2000).

An unserem Beispiel wird sichtbar, dass citizenship sich nicht in politischen und sozialen Partizipationsrechten und -pflichten erschöpft und auch nicht unmittelbar aus diesen abzuleiten ist. Die Verwirklichung der politischen und sozialen Dimensionen des Bürger-Werdens und -Seins setzen die Möglichkeit kultureller Teilhabe voraus. Eine wesentliche Rolle in dem vierdimensionalen Prozess citizenship spielen die Medien und 
insbesondere das Fernsehen. Jostein Gripsrud (1999: 2) beschreibt die heutige Bedeutung des Fernsehens als „primary source of common knowledge“, „a widely shared pool of information and perspectives from which people shape their conceptions of self, world and citizenship “. Identität als StaatsbürgerIn wird damit nicht nur und vermutlich nicht einmal vorrangig durch politische Partizipationsmöglichkeiten erworben, sondern gleichermaßen durch die diskursiven Verhandlungen der eigenen kulturellen Praktiken.

Die von uns entwickelte Definition von cultural citizenship geht bewusst nicht von Ansprüchen und Rechten als dem Kern von citizenship aus. Damit ist sie in ihrem Ursprung nicht normativ angelegt. Zwar legitimieren Ansprüche und Rechte jene kulturellen Praktiken, die konsensbildend wirken, zugleich sind sie aber nur Ausdruck eines umfassenderen Prozesses, in dem citizenship zugewiesen und ausgeübt wird. Murdock (1999: 11f.; zuerst 1994: 158f.) hat ausgeführt, in welcher Weise Rechte und Ansprüche einer cultural citizenship in der Mediengesellschaft als Voraussetzung für den Entwurf eigener kultureller Identitäten präzisiert werden können. Danach beinhaltet cultural citizenship als kompetente Teilhabe an den kulturellen Ressourcen einer Gesellschaft folgende - letztlich an Medien gerichtete - Ansprüche7

- Ansprüche auf Informationen, um Grundlagen für politische und soziale Entscheidungen zu besitzen. Traditionell galt dies in den Medien als Domäne der nicht-fiktionalen Medienangebote.

- Ansprüche auf Erfahrungen, die die Vielfalt möglicher Lebensweisen zum Ausdruck bringen und somit Ideen für eigene Identitätskonzepte liefern. Traditionell galt dies in den Medien als Domäne der fiktionalen oder als „unterhaltend“ bezeichneten Produktionen.

- Ansprüche auf Wissen, als Möglichkeit, Informationen und Erfahrungen sinnvoll zu interpretieren und zu integrieren. Das erfordert Zugang zu Erklärungsansätzen, die das Allgemeine mit dem Besonderen, das Aktuelle mit dem historisch Gewachsenen, die Mikroebene mit der Makroebene verbinden. Bezogen auf Medien verweist das auf Angebote, die die traditionelle Scheidung von „U“- und „E“- (bzw. „I“-)Angeboten durchbrechen.

- Schließlich Ansprüche auf Teilhabe, die den Mitgliedern des vermeintlich passiven und als uniforme Masse gedachten Publikums eine Stimme verleihen und ihren aktiven Anteil am Ringen um Bedeutungen in der Mediengesellschaft zum Ausdruck bringen. Im Medienprozess verweist das auf die Notwendigkeit einer konsequenten Zuwendung zum Publikum, also auf eine stärkere Orientierung an den Interessen der Zuschauenden wie auch ihre stärkere Einbeziehung in die Programmgestaltung.

Mit Murdocks Unterscheidung von vier Ansprüchen wird eine weiter gehende Ausdifferenzierung der zuerst von Hegel (Phänomenologie des Geistes, 1807) entwickelten Unterscheidung zwischen Wissen und Erfahrung vorgenommen. Diese Unterscheidung spielte für die Entwicklung des modernen Journalismus, insbesondere des recherchierenden Journalismus in der Tradition der Chicago School of Sociology, von Anfang an eine zentrale Rolle (vgl. Lindner 1990: 60). Charakteristisch für die aktuelle, als Mediengesellschaft bezeichnete Formation ist, dass die Ansprüche auf Erfahrung und Teil-

7 Murdock spricht von „rights“. Wir haben uns für die Übersetzung als „Ansprüche“ statt „Rechte“ entschieden, da erstere im Unterschied zu letzteren weniger mit einklagbaren, formalisierten gesetzlichen Regelungen assoziiert werden. Die von Murdock genannten Ansprüche richten sich u. a. als Erwartungen an die Medien und die Medienschaffenden. 
habe im medialen Diskurs deutlich an Relevanz gewonnen haben, während die Ansprüche auf Information und Wissen - als geradezu paradigmatische Leistungen des öffentlich-rechtlichen Rundfunks - an Relevanz verloren haben. Diese Relevanzverschiebung lässt sich im ersten Schritt an einer veränderten Angebotsstruktur der Medien festmachen. So haben partizipative Unterhaltungsangebote in Umfang und Variationsreichtum gegenüber traditionellen Informationsangeboten deutlich zugenommen. Doch nicht allein quantitative Veränderungen markieren diesen Wandel. Vor allem der mediale Diskurs um die neuartigen Medienangebote, die an Erfahrung und Teilhabe orientiert sind, ist Ausdruck der gesellschaftlichen Bedeutungsveränderung. ${ }^{8}$

Erfahrung und Teilhabe stellen damit nicht einen Ersatz für Information und Wissen dar, vielmehr werden sie zu sich komplementär ergänzenden Bestandteilen der Mediengesellschaft. Information wird erst auf der Basis von Erfahrung zu relevantem Wissen. Partialisierte Informationen ohne lebensweltlichen Bezug verlieren so an gesellschaftlicher Bedeutung. Auch wenn dieser Prozess in erster Linie durch ökonomische Entwicklungen und kommerzielle Interessen induziert wurde, so beschreibt er dennoch keineswegs automatisch eine Form der De- oder Entpolitisierung. Auf der Ebene von Politik und Öffentlichkeit wirkt sich der Verlust an Glaubwürdigkeit und Bindungskraft traditioneller politischer Institutionen vielmehr zugunsten der diversen alternativen sozialen Gruppen aus, die unter den Stichworten „neue soziale Bewegungen“, „non-governmental organisations“ (NGOs) oder „Zivilgesellschaft" firmieren. Auf der Ebene der Massenmedien beinhaltet diese Entwicklung eine Verabschiedung vom paternalistischen Informationsanspruch des Public Service-Konzeptes zugunsten einer Ankopplung von mediengebundenen Informationen an die Erfahrungswelt des Publikums.

Diese Überlegungen wollen wir im Weiteren vertiefen. Dabei soll das theoretische Konzept cultural citizenship an zwei gleichermaßen aktuellen, aber sehr unterschiedlichen Problemfeldern exemplarisch auf seine Reichweite, Leistungsfähigkeit und Bedeutung für die Medienforschung und Kommunikationswissenschaft hin erprobt werden. Zum einen wollen wir im Bereich aktueller Entwicklungen des Fernsehjournalismus und -dokumentarismus Phänomene der Veralltäglichung von Medieninhalten und Rezeptionsweisen betrachten. Hier erweist sich cultural citizenship als theoretische Folie zur Analyse von Mikroprozessen, die die Bedeutungskonstruktion und Bedeutungszuweisung im Rahmen der Medienrezeption umfassen.

Dass das Konzept cultural citizenship nicht auf die Analyse von Mikroprozessen beschränkt bleibt, wollen wir zum anderen am Beispiel der UN-Konferenz „World Summit on the Information Society“ (WSIS) zeigen. In der Vorbereitung und der Durchführung der Weltkonferenz wurde und wird die Relevanz der kulturellen Staatsbürgerschaft im Kontext medialer Globalisierungsprozesse erörtert. Im Mittelpunkt stehen in dieser Debatte Makroprozesse der massenmedialen Kommunikation. Die politischen und ökonomischen Bedingungen der globalen Mediengesellschaft rücken in diesem Beispiel gegenüber den vorher fokussierten individuellen und gruppenbezogenen Rezeptionsweisen in den Vordergrund. Das Verhältnis von Zivilgesellschaft(en), Regierungen und wirtschaftlichen Kräften gewinnt im Kontext internationaler Kommunikationsprozesse neue Kontur. In diesem Zusammenhang zeigt sich besonders deutlich, dass cultural citizenship auch eine normative Seite besitzt, die über die vorher gelieferte analyti-

8 Vgl. exemplarisch die Diskurse um „Big Brother“, etwa in Mikos u. a. (2000) oder in Hill und Palmer (2002). 
sche Definition hinausreicht und Grundrechte auf kommunikative und kulturelle Teilhabe begründen kann. Die beiden hier gewählten Beispiele sollen skizzenhaft die Brauchbarkeit des theoretischen Konzeptes sichtbar machen. Da sie unterschiedlichen wissenschaftlichen (Reality TV) und politischen (WSIS) Diskursen entstammen, argumentieren wir in den Abschnitten 5 und 6 auf je unterschiedlichem Abstraktionsniveau.

\section{Cultural Citizenship und Medienalltag - Phänomene der Entgrenzung}

Der Boom des Reality TV und seine Ausdifferenzierung in verschiedene Sub-Genres (vgl. Klaus/Lücke 2003) bringt zwei zentrale Trends in der Entwicklung des Fernsehens zum Ausdruck: Faktizität bestimmt zunehmend die Fernsehunterhaltung und gleichzeitig werden Fernsehjournalismus und -dokumentarismus verstärkt durch Regeln des Fiktionalen geprägt. Fakt und Fiktion, Journalismus und Unterhaltung, Information und Erfahrung - diese Phänomene sind zunehmend nicht mehr sauber voneinander getrennt, sondern treten in Misch- und Hybridformen in Erscheinung (vgl. Lünenborg 2004). „Performatives Realitätsfernsehen“ (Keppler 1994), „Affektfernsehen“ (Bente/Fromm 1997), „factual entertainment“ (Brunsdon et al. 2001) sind Begriffe, mit denen KommunikationswissenschaftlerInnen die Veränderungen zu fassen versuchen. Im Kontext einer Diskussion von cultural citizenship ist die Entwicklung des Fernsehens durch eine Veränderung des vielen Sendungen zugrunde liegenden Informationsbegriffs gekennzeichnet. Der Informationsbegriff erschöpft sich dabei nicht (länger) in der bloßen Vermittlung isolierter Fakten, deren Relevanz allein von den KommunikatorInnen festgelegt wird. Er entwickelt sich vielmehr im Sinne Murdocks (1999) hin zu einem erfahrungsbasierten Verständnis, das die prinzipielle Möglichkeit zur Teilhabe einschließt. Die unter der Bezeichnung Reality TV zusammengefassten Genres (Reality Magazin, Docu-Soap, Real-Life-Soap) sind insbesondere durch vier Merkmale charakterisiert ${ }^{9}$ :

1. Alltag und Alltagserfabrung werden zum zentralen Bestandteil neuer Medienangebote. Die Veralltäglichung von Medieninhalten steht in Wechselwirkung zu der Alltagskontextuierung der Rezeptionssituation. Mit dem Begriff Glocalisation wird treffend beschrieben, wie globale Phänomene der gesellschaftlichen Entwicklung im Fernsehen in lokalem, alltagsnahem Handeln ihren Ausdruck finden und auf diese Weise ihr Publikum erreichen (vgl. Morley 1992). Narrowcasting durch Fernsehen wird damit nicht (allein) durch veränderte technische Übertragungswege erzielt, sondern über ein „Näherrücken“ der Medieninhalte durch ihr Anknüpfen an die unmittelbaren Lebenserfahrungen des Publikums (vgl. Mikos 2001; Fiske 1994). In Murdocks Terminologie lassen sich diese Phänomene wie folgt beschreiben: Ansprüche auf Teilhabe und Erfahrung rücken in den neueren Medienangeboten gleichgewichtig neben jene nach Information und Wissen.

9 Die hier nur knapp dargestellten Überlegungen zu Veränderungen des aktuellen Fernsehjournalismus sind Bestandteil des DFG-finanzierten Habilitationsprojektes von Margreth Lünenborg. Unter dem Titel „Kulturelle Bedeutungsproduktion durch Journalismus. Die Integration der Publikumsperspektive in die Journalistik“ entwickelt das Projekt theoretische Zugänge, den Cultural Studies Approach für die Journalistik zu nutzen, und beschäftigt sich auf dieser Grundlage empirisch mit Docu-Soaps als neuer Form des seriellen, narrativen Fernsehdokumentarismus. Eine aktuelle Definition des Reality TV und eine Analyse seiner Subgenres liefern Elisabeth Klaus und Stephanie Lücke (2003). 
2. Alltagsmenschen in ibrer jeweiligen sozialen und kulturellen Kontextuierung werden zu ProtagonistInnen neuer Fernsehformate. Im Unterschied zu der Public Servicedominierten Phase des Fernsehens sind es nicht mehr vorrangig die politischen und wirtschaftlichen Eliten, die mit den publizistischen Eliten via Fernsehen ins Gespräch kommen. Die Partizipation von Alltagsmenschen in Talkshows, Game- und Quizshows, aber auch in Genres des Reality TV bricht diesen Diskurs der Eliten auf. Gerade diese Veränderung des televisuellen Personals beendet unumkehrbar den paternalistischen Gestus öffentlich-rechtlichen Bildungs- und Informationsfernsehens. Durch die stärkere Laienorientierung und die größere Bedeutung populären Wissens wird das Publikum als Konsument und politisches Subjekt zum Mitträger des Programmes. Der dänische Medienwissenschaftler Bondebjerg (1996: 29) bezeichnet diese Veränderung als „democratization of an old public service discourse, dominated by experts and a very official kind of talk, and the creation of a new mixed public sphere, where common knowledge and everyday experience play a much larger role“. Letztlich stellt die damit einhergehende engere Verknüpfung von Informationen und Erfahrungen die Voraussetzung für die Wissensproduktion im Sinne Murdocks bereit.

3. Privatheit und Öffentlichkeit werden zu komplementären Bestandteilen des medialen Diskurses. Privatisierung, Intimisierung und Personalisierung sind zentrale Charakteristika der neuen Fernsehgenres. Diese Integration privater Diskurse in die medienöffentliche Auseinandersetzung zeigt, dass sich die traditionellen, so klar wie starr erscheinenden Grenzen zwischen Privatheit und Öffentlichkeit auflösen. Authentizität wird zu einem zentralen Qualitätsmaßstab des neuen Fernsehens. Dabei ist Authentizität kein Merkmal, das einem Medientext eigen ist. Sie wird vielmehr vom Publikum dem Medientext im Prozess der Rezeption zugewiesen (vgl. Lünenborg 2002: 183). Authentizität gewinnen Charaktere in den Genres des Reality TV für das Publikum dann, wenn es ihnen gelingt, öffentliche Diskurse in die allägliche Lebenswelt zu übertragen und ihnen in der Privatsphäre Relevanz zu verleihen. Eine Trennung von Öffentlichkeit und Privatheit als antagonistische Sphären erscheint vor diesem Hintergrund nicht länger angemessen, vielmehr entstehen gesellschaftlich relevante Diskurse im Zusammenwirken intimer und öffentlicher Prozesse (vgl. versch. Beiträge in: Hermann/Lünenborg 2001).

4. Fakt und Fiktion sind nicht eindeutig als unterschiedliche mediale Wirklichkeitsmodi zu identifizieren. Den souveränen Umgang des Publikums mit dem angeeigneten Wissen über mediale Konstruktionsprozesse voraussetzend agieren aktuelle Fernsehformate an der Grenze zwischen Fakt und Fiktion und überqueren diese Grenze beständig (vgl. Klaus/Lünenborg 2002). Durch die (tendenzielle) Auflösung dieser für die Fernsehproduktion lange Zeit formativen Unterscheidung verliert ein Teil des Medienangebots seinen spezifischen Wahrheitsanspruch. Fakt und Fiktion, die beiden „regimes of truth“ wie Hartley (1992) die konkurrierenden Interpretationsmodi bezeichnet, verhalten sich nicht länger hierarchisch zueinander. Ein dramatisches Beispiel dafür lieferte der Irakkrieg 2003, dessen mediale Präsentationsstrategien mit dem Bericht über ein Fußballspiel verglichen wurden (vgl. Hoff 2003; Leder 2003). Heute zeigt sich immer deutlicher, dass letztlich das Publikum dem jeweiligen Medientext Glaubwürdigkeit im Sinne eines faktischen oder emotionalen Realismus zuweisen muss (vgl. Ang 1986; Lünenborg 2002). Die von Murdock mit cultural citizenship verbundenen Ansprüche auf Wissen und Teilhabe werden damit zugleich als Zwang wie als Möglichkeit sichtbar, an der Neuaushandlung der Grenzen von Öffentlichkeit und Privatheit, von Fakt und Fiktion mitzuwirken. 
Die Bedeutung und Entwicklung des Reality TV spiegelt die gewachsene Bedeutung der kulturellen Identitätsfindung durch Medien. Cultural citizenship manifestiert sich in den und durch die Genres des Reality TV, indem eigene Erfahrungen mit den medial vermittelten abgeglichen werden können. Zu untersuchen bleibt, welche Formen der cultural citizenship dadurch entstehen und welche Einschlüsse und Ausschlüsse durch die so fundierte Bedeutungsproduktion vorgenommen werden. Den Anspruch auf Teilhabe kann das Publikum dort realisieren, wo Bezüge zur eigenen Lebenswirklichkeit sichtbar werden. Dabei geht es nicht etwa um bloße Reproduktion des selbst Erlebten. Livingstone $(1999,96 \mathrm{ff}$.) hat darauf hingewiesen, dass medial vermitteltes Wissen durch die Kopplung von Erfahrung und Information geschaffen wird. Dabei unterscheidet sie zwischen zwei grundsätzlich verschiedenen Verknüpfungsformen: einerseits „recognition“ als Wiedererkennen des schon Bekannten, bereits Vertrauten, zuvor Erfahrenen; andererseits „discovery“ als Entdeckung des Neuen, noch-nicht-Erfahrenen, bisher nicht Gewussten. In solchem Wissen wird Weltbezug verwirklicht und ermöglicht. Ansprüche auf Teilhabe setzen voraus, dass die vielfältigen Publika in den Medienangeboten sowohl ihre Erfahrungen wiedererkennen als auch neue Erfahrungen machen können. Medien müssen dann sowohl „recognition“ als auch „discovery“ ermöglichen. Der Herausbildung einer umfassenden citizenship wäre in der Konsequenz am ehesten gedient „by open programmes that offer a diversity of positions and require the engaged participation of viewers“ (Murdock 1999: 13).

Weil die verschiedenen Publika als Adressaten der Fernsehprogramme sprachräumlich bzw. nationalstaatlich verortet sind, werden mit der Betrachtung der aktuellen Fernsehentwicklung zahlreiche Fragen auf der Mikroebene des Medienprozesses aufgeworfen. Mit unserem zweiten Beispiel, das den WSIS-Prozess skizziert, wollen wir uns der Makroebene der Medienentwicklung zuwenden. Dabei betrachten wir cultural citizenship im Kontext des Globalisierungsprozesses. Uns interessiert an dem Beispiel vor allem, welche Rolle Medien und Kommunikation im Konzept des Weltbürgers und den damit verbundenen Forderungen nach sozialer, politischer, individueller und kultureller Gleichberechtigung spielen.

\section{Cultural Citizenship und Weltinformationsgesellschaft}

Die Frage der Konstitution von citizenship unter den Bedingungen von Globalisierung, technologischem Wandel, weltweitem Medientransfer und global operierenden Medienkonzernen wurde intensiv bei der Vorbereitung und in der Durchführung des „World Summit on the Information Society“ (WSIS) der Vereinten Nationen diskutiert, dessen erster Teil vom 10. bis 12. Dezember 2003 in Genf stattfand und dessen zweiter Teil für 2005 in Tunis geplant ist. ${ }^{10}$ Überraschend ist, wie wenig diese UN-Weltkonferenz bisher von der deutschen Öffentlichkeit allgemein und der Kommunikationswissenschaft insbesondere überhaupt wahrgenommen worden ist. ${ }^{11}$ Dabei berührt sie grundlegende Fragen der zukünftigen Entwicklung von nationalen wie internationalen Medienstrukturen, Medienangeboten und Kommunikationsweisen, die gleichermaßen auch auf der wissenschaftlichen Agenda stehen.

10 Auf der Website (www.itu.int) der die Konferenz ausrichtenden „International Telecommunications Union" finden sich alle offiziellen Dokumente.

11 Demgegenüber war das ECCR aktiv in die Vorbereitungen involviert, und auch im IAMCRNewsletter fanden sich dazu Diskussionsbeiträge. 
Die Aufgabe des WSIS ist zu klären, wie eine Teilhabe aller Nationen und Staaten an den Entwicklungen der Informations- und Kommunikationstechnologien Wirklichkeit werden könnte und entsprechende Maßnahmen dazu zu beschließen. Zentral ist also die Frage der Verhandlung von Ein- bzw. Ausschlüssen in der internationalen Staatengemeinschaft mittels massenmedialer Kommunikation. In der Verwirklichung dieses Zieles standen sich von Anfang an zwei Visionen gegenüber, die durch die AkteurInnen der Wirtschaft auf der einen Seite und der „Zivilgesellschaft“ auf der anderen Seite repräsentiert werden. Die Wirtschaft setzt vor allem auf den Ausbau der Infrastruktur (vgl. Capdevilla 2003a). Ausdruck davon war das zunächst auch von zahlreichen Regierungen mitgetragene Ziel, dass jedes Dorf der Welt im Jahr 2010 über einen Internetanschluss verfügen solle (Capdevilla 2003b). Die andere Vision thematisiert demgegenüber Ausschlussprozesse, Macht und Medienkonzentration, Demokratie und kulturelle Verschiedenheit als Rahmenbedingungen der Teilhabe an der Weltinformationsgesellschaft. Den Ausgangspunkt für Diskussionen über die Weltinformationsgesellschaft sollen demzufolge nicht infrastrukturelle Entwicklungen bilden, sondern die „Vision of an Information Society beneficial to all“ (Bucharest 2002) und damit Menschenrechte sowie soziale, politische und kulturelle Entwicklungschancen. Nachdem zunächst beide Perspektiven unvereinbar erschienen, wurden schließlich in Genf doch noch zwei Dokumente verabschiedet, die gemeinsame Prinzipien zum Aufbau der Weltinformationsund Kommunikationsgesellschaft enthalten (Declaration of Principles, WSIS 2003b) und entsprechende Schritte dazu formulieren (Plan of Action, WSIS 2003c).

Die Relevanz von cultural citizenship zeigt sich im WSIS-Prozess ${ }^{12}$ auf doppelte Weise. Zum einen kommt sie auf der Ebene der AkteurInnen der Konferenz zum Ausdruck, zum anderen in den dort verhandelten Inhalten.

Zunächst zur Akteursebene: Die Nicht-Regierungsorganisationen (NGOs) ${ }^{13}$ formierten sich im Vorfeld des World Summits und brachten ihre Forderungen und Ansprüche gegenüber den Regierungs- und Wirtschaftsvertretern ein. Bei der zweiten UNVorbereitungskonferenz im Februar 2003 wurde diesen heterogenen Gruppen unter dem Dach „Zivilgesellschaft“ erstmals ein offizieller Platz bei einem UN-Weltgipfel eingeräumt (vgl. Capdevilla 2003b). Dabei zählen zur „Zivilgesellschaft“ national oder regional verankerte Gruppen, beispielsweise jene, die für die Rechte indigener Völker eintreten, ebenso wie transnationale Gruppierungen, die sich für den Umweltschutz oder die Emanzipation von Frauen (vgl. APC Women 2003) engagieren. Dass ihr Anspruch, an den Entscheidungen über die internationale Medien- und Kommunikationsentwicklungen teilzuhaben, erfolgreich war, zeugt einerseits vom Bedeutungsverlust nationalstaatlich legitimierter Interessenvertretung und andererseits von der Bedeutung der Medien, insbesondere dem Internet, im globalen politischen Raum. Per Newsletter, Websites und Online-Konferenzen ${ }^{14}$ wurden im Internet in Abgrenzung zu den offiziellen Dokumenten Positionen ausgetauscht und Forderungen diskutiert, die den verschiedenen, im Vergleich zu den Regierungen marginalen Gruppen letztlich das gemeinsame, erfolgreiche Agieren ermöglichten. Zahlreiche Positionen der Zivilgesellschaft zum

12 Den Prozesscharakter des World Summit on the Information Society hat Hans Klein (2003) analysiert.

13 Im Juli 2002 trafen sich über 1.000 NGOs aus mehr als 100 Ländern zum World Civil Society Forum, das der Vorbereitung des WSIS gewidmet war (WSIS 2002a, 2002b).

14 So initiierte die UNESCO von Dezember 2002 bis Mitte Januar 2003 ein Online-Forum, dessen Ergebnisse wiederum im Internet nachzulesen sind (UNESCO 2003). 
Weltinformations-Gipfel, die diese in Vorbereitung der Konferenz entwickelten, finden sich in den Genfer Schlussdokumenten wieder.

Zur inhaltlichen Ebene: Mit der Teilhabe der Zivilgesellschaft wurde cultural citizenship auch auf der Ebene der im WSIS-Prozess diskutierten Inhalte bedeutsam. In den Vorbereitungsdokumenten der NGOs finden sich Ausführungen und Forderungen, die alle Dimensionen von citizenship ansprechen (vgl. Civil Society Coordination Group 2002; WSIS 2002a, 2002b, 2003a). Civil, political und social citizenship werden gleichberechtigt behandelt. In dem zur zweiten UN-Vorbereitungskonferenz vorgelegten Papier wird beispielsweise der Zugang zu den Informations- und Kommunikationstechnologien unabhängig von nationaler und ethnischer Zugehörigkeit, ökonomischem Reichtum und Geschlecht gefordert (WSIS 2003a). Hier wird insbesondere auch auf die Notwendigkeit von Public Service-Medien hingewiesen. Ein eigener Abschnitt ist der Sicherung von „Cultural identity and linguistic diversity, local content und media development" gewidmet. Darin reichen die Ziele von der Unterstützung lokaler Community-Medien über die Sicherung der Vielsprachigkeit im Cyberspace bis hin zum Schutz gegen eine unfaire Verwendung indigenen Wissens und intellektuellen Eigentums (ebd.: Teil 8). Cultural citizenship ist hier direkt angesprochen als Möglichkeit, eigene kulturelle Praktiken wie etwa die Sprache in den Prozess der Gestaltung der Weltinformationsgesellschaft einbringen zu können (vgl. dazu auch UNESCO/ WSIS 2002: 4).

Die Debatten rund um die UN-Weltkonferenz über die Informationsgesellschaft lassen sich entlang dem hier entwickelten Begriff cultural citizenship strukturieren: Im Zentrum stand und steht dabei die Forderung, die gleichberechtigte Zugehörigkeit zur weltweiten Informations-, Kommunikations- oder Mediengesellschaft zu ermöglichen. Ein solcher Anspruch reicht weit über technologische Partizipation hinaus und fokussiert die Anerkennung eigener kultureller Bedeutungspraxen. Citizenship ist als Selbst- und Fremdzuschreibung in all seinen Dimensionen nur auf der Basis der sprachlich, historisch, sozio-strukturell und territorial vermittelten Gemeinschaften zu erzielen. Im Sinne von Murdocks Typologie finden sich in der in Genf schließlich verabschiedeten „Declaration of Principles“ (WSIS 2003b) alle mit cultural citizenship verbundenen Ansprüche auf Teilhabe - auf das Einbringen eigener Erfahrungen in die bevorstehenden Entwicklungen, auf Informationen und ein Wissen, das beide Bereiche verknüpft. Die von Murdock formulierten Ansprüche können nicht isoliert, sondern nur im Zusammenspiel cultural citizenship begründen. Informationen sind notwendig an Erfahrungen gebunden, um gemeinsam Wissen zu schaffen und kulturelle Teilhabe zu ermöglichen. Sie beinhalten die medienvermittelte Möglichkeit, sowohl zu den symbolischen Ressourcen der Gesellschaft beizutragen als auch an diesen gleichberechtigt zu partizipieren.

In diesem Zusammenhang steht die zunehmend lauter werdende Forderung nach der internationalen Verankerung von Kommunikationsrechten. Beispielsweise hat Cees Hamelink (2002) in seinem streitbaren Aufsatz „Moral challenges in the information society“ darauf aufmerksam gemacht, dass mehr Informationen allein keinen Fortschritt in Bezug auf die gleichberechtigte Teilhabe aller Menschen an den Informations- und Kommunikationstechnologien bedeute. Vielmehr käme es darauf an, wie Informationen genutzt, eingebettet und diskutiert würden, wer Entscheidungen darüber in wessen Interesse fälle und für Ergebnisse verantwortlich sei. Der Begriff der Weltinformationsgesellschaft verstärke die verbreiteten Mythen über die sich vermeintlich automatisch ergebenden aufklärerischen Wirkungen eines „Mehr“ an Informationen (vgl. auch WACC 2002). Die Welt brauche nicht Informations- bzw. Wissensgesellschaften, sondern 
Kommunikationsgesellschaften, um die sozialen, ökonomischen, politischen und ökologischen Anforderungen zu bewältigen. Hamelink fordert die Verankerung eines „Rechtes auf Kommunikation“ als Menschenrecht, das neben Informationsrechten auch Rechte auf den Schutz der Privatsphäre und auf Teilhabe beinhaltet sowie kulturelle Rechte einschließt. ${ }^{15}$

Hartley vertritt die Position, dass die Formulierung von Rechten an eine späte formative Phase der jeweiligen Aspekte von „Staatsbürgerschaft“ geknüpft ist. Demnach ist cultural citizenship „in the process of formation - being made to mean something long before it can be institutionalized and legislated. In my view ,cultural citizenship“ is at a late stage of rights-formation, moving into formal legislative existence in a number of contexts" (Hartley 1999: 161). Insgesamt scheint der WSIS-Prozess diese Position zu bestätigen.

\section{Resümee}

Gerade weil unsere Beispiele so unterschiedliche Prozesse beleuchten, zeigt sich, dass das Konzept cultural citizenship dazu beitragen kann, bislang voneinander getrennt beobachtete Phänomene innerhalb der Kommunikationswissenschaft zueinander in Beziehung zu setzen. Cultural citizenship macht sichtbar, dass Mikro- und Makroprozesse der Integrationsleistung von Medien sich gegenseitig durchdringen, und fordert damit eine integrative Betrachtung individueller Bedeutungsproduktion und staatenübergreifender Kommunikationsprozesse ein. Individuelle und kollektive Identitätsbildung, Verfahren der Inklusion oder Exklusion sind maßgeblich medial konstituiert. Das Konzept erlaubt es weiter, eine Brücke zwischen jenen, lange als „natürlich“ erscheinenden Dualismen zu schlagen, die zunehmend komplementär wirksam werden. Öffentlichkeit und Privatheit, Politik und Personalisierung, Unterhaltung und Information, Emotio und Ratio, Fakt und Fiktion, Inszenierung und Dokumentation, diese Begriffspaare sind prekäre Trennungen geworden. In aktuellen gesellschaftlichen Diskursen werden sie immer stärker verzahnt. Initiator und Träger dieser Entwicklung sind die Medien. Um diese neuen Formen der Kommunikation zu verstehen, greift eine kulturkritische Position zu kurz, mystifiziert sie doch selber die vermeintlichen Dualismen. ${ }^{16}$ Ebenso wenig kann sich die Kommunikationswissenschaft damit begnügen, nur empirische Beschreibungen und somit eine allein affirmative Begleitung der Entwicklungen vorzunehmen, ohne nach ihrer Bedeutung und den Konsequenzen für die soziale Struktur der Gesellschaft zu fragen.

Wir halten das Konzept cultural citizenship für geeignet, diese sich auf allen Ebenen des Medienprozesses vollziehenden Konvergenz- und Entgrenzungsphänomene besser $\mathrm{zu}$ verstehen und in ihrem Gesellschaftsbezug zu untersuchen. Die damit vorgenommene Erweiterung der verschiedenen Dimensionen von Staatsbürgerschaft (citizenship)

15 Weiter ausgearbeitet und diskutiert wird die Idee eines Rechts auf Kommunikation als Menschenrecht auf der Website der „CRIS Campaign“ (Communication Rights in the Information Society, www.crisinfo.org), ein Zusammenschluss verschiedener NGOs (CRIS Campaign 2003).

16 An der feministischen Debatte um Habermas' Konzept von Öffentlichkeit als Gegenpol zur Privatheit, ist das exemplarisch deutlich geworden (vgl. dazu Fraser 1989: 173-221; Hermann/Lünenborg 2001). 
hilft, die Bedeutung von Medien gleichermaßen für den Prozess der Herausbildung individueller und subkultureller Identitäten wie auch die Konstituierung von Öffentlichkeiten analytisch zu erfassen. Sie ermöglicht damit die notwendige theoretische Verbindung der politischen Ideen der Aufklärung mit einer Kritik an den daran geknüpften Ausgrenzungen (etwa entlang von Gender und Ethnie), unzulässigen Verallgemeinerungen und immanenten Dichotomisierungen. Cultural Citizenship wird mit, durch und mittels Medien erworben.

Im Mittelpunkt der Diskussionen um Reality TV und Weltinformationsgesellschaft steht eine Neuformulierung des Informationsbegriffs. Sowohl auf der Mikroebene der veränderten Medienangebote wie auf der Makroebene des Bemühens um die gleichberechtigte Teilhabe aller Staaten und Völker an den neuen Informations- und Kommunikationstechnologien wird der Wert von Informationen und Erfahrungen verhandelt, werden Ansprüche an Wissen und Teilhabe definiert, die die kompetente Teilhabe an den kulturellen Ressourcen der Gesellschaft ermöglichen. Deshalb kann cultural citizenship als Interpretationsscharnier zwischen individualisierter alltäglicher Medienaneignung und politisch konstituierter Medienöffentlichkeit dienen. Cultural citizenship bietet den theoretischen Kontext, um Verbindungen herzustellen zwischen Information und Erfahrung, zwischen Ansprüchen auf Differenz und dem Recht auf Gleichberechtigung, schließlich auch zwischen den Agenden der Zivilgesellschaft und der klassischen nationalstaatlichen AkteurInnen. Indem das Konzept eine Brücke schlägt zwischen den mit solchen Fragen befassten Mikro- und Makroanalysen und zwischen Rezeptionsforschung und kritischer Kulturanalyse, kann es dazu beitragen, die gegenwärtige Medienentwicklung mit ihren Entgrenzungen und neuen Grenzsetzungen besser zu verstehen und angemessener empirisch zu erfassen.

\section{Literatur}

Ang, Ien (1986): Das Gefübl Dallas. Zur Produktion des Trivialen. Bielefeld: Deadalus.

Ang, Ien (1998): Desperately Seeking the Audience. London/ New York: Routledge.

APC Women (2003): Information and Communication Technologies: A Women's Agenda. www.apcwomen.org/work/policy/women-rights.html [23. 3. 2003].

Bente, Gary/Bettina Fromm (1997): Affektfernseben. Motive, Angebotsweisen und Wirkungen. Opladen: Leske + Budrich (Schriftenreihe Medienforschung der Landesanstalt für Rundfunk NRW; Bd. 24).

Bondebjerg, Ib (1996): Public discourse/private fascination: Hybridization in „true-life-story' genres“. In: Media, Culture and Society, 18. Jg., S. 27-45.

Brunsdon, Charlotte u. a. (2001): Factual Entertainment on British Television. The Midlands TV Research Group's 8-9 Project. In: European Journal of Cultural Studies, 4. Jg., Heft 1, S. 29-62.

Bucharest (2002): The Bucharest Pan-European Conference in Preparation of the World Summit on the Information Society: Towards an Information Society: Principles, Strategy and Priorities for Action. 9. November 2002. www.crisinfo.org [26.3.2003].

Capdevila, Gustavo (2003a): Summit Prep meet caught between two visions. www.eccr.info [17.2.2003].

Capdevila, Gustavo (2003b): Civil Society wins a place at WSIS table. www.eccr.info [28. 2. 2003].

Civil Society Coordination Group (2002): Statement to the Informal Meeting on Content \& Themes Geneva, 16-18 September 2002. www.itu.int/wsis/documents [11.2.2003].

CRIS Campaign (2003): Whose Information Society? www.crisinfo.org [26.3.2003].

Dahrendorf, Ralf (1957): Soziale Klassen und Klassenkonflikt in der industriellen Gesellschaft. Stuttgart: Enke.

Dörner, Andreas (2000): Politische Kultur und Medienunterhaltung. Zur Inszenierung politischer Identitäten in der amerikanischen Film- und Fernsehwelt. Konstanz: UVK. 
Dörner, Andreas (2001): Politainment. Politik in der medialen Erlebnisgesellschaft. Frankfurt a. M.: Suhrkamp.

Du Gay, Paul/Stuart Hall u.a. (1996): Doing Cultural Studies. The Story of the Sony Walkman. London: Sage.

Fiske, John (1994): Media Matters. Everyday Culture and Political Change. Minneapolis/London: University of Minnesota Press.

Fiske, John (2001): Understanding Popular Culture. 8. Auflage. London/New York: Routledge.

Fraser, Nancy (1989): Widerspenstige Praktiken. Macht, Diskurs, Geschlecht. Frankfurt a. M.: Suhrkamp.

Fraser, Nancy/Linda Gordon (1994): Civil Citizenship against Social Citizenship? On the Ideology of Contract-Versus-Charity. In: Bart van Steenbergen (Hrsg.): The Condition of Citizenship. London/Thousand Oaks/New Delhi: Sage, S. 90-107.

Gripsrud, Jostein (Hrsg.) (1999): Television and Common Knowledge. London/New York: Routledge.

Grossberg, Lawrence (1999): Der Cross Roads Blues der Cultural Studies. In: Andreas Hepp, Rainer Winter (Hrsg.): Kultur - Medien - Macht. Cultural Studies und Medienanalyse. 2., überarbeitete und erweiterte Auflage. Opladen/Wiesbaden: Westdeutscher Verlag, S. 15-32.

Hamelink, Cees J. (2002): Moral challenges in the Information Society. www.wacc.org.uk/publications/md/md2002-4/hamelink.html [17. 12. 2002].

Hartley, John (1992): Regimes of Truth and the Politics of Reading: A Blivit. In: John Hartley (Hrsg.): Tele-ology. London/New York. Routledge, S. 45-63.

Hartley, John (1996): Popular Reality. Journalism, Modernity, Popular Culture. London: Edward Arnold.

Hartley, John (1999): Uses of Television. London/New York: Routledge.

Hepp, Andreas/Rainer Winter (Hrsg.) (1999): Kultur - Medien - Macht. Cultural studies und Medienanalyse. 2., überarbeitete und erweiterte Auflage. Opladen/Wiesbaden: Westdeutscher Verlag.

Hermann, Friederike/Margreth Lünenborg (Hrsg.) (2001): Tabubruch als Programm. Privates und Intimes in den Medien. Opladen: Leske + Budrich.

Hermes, Joke (1998): Cultural Citizenship and Popular Fiction. In: Kees Brants, Joke Hermes und Liesbet van Zonen (Hrsg.): The Media in Question. Popular Cultures and Public Interests. London/Thousand Oaks/New Delhi: Sage, S. 156-167.

Hill, Annette; Palmer, Garreth (eds.) (2002): Special Issue Big Brother. In: Television \& New Media, 3, 3, S. 251-340.

Hoff, Hans (2003): „Goal in Bagdad“ mit CNN an der Front. Der US-Nachrichtenkanal berichtet im Stil der Sportreportage. In: Süddeutsche Zeitung vom 24.3.2003, S. 21.

Johnson, Richard (1985): Was ist überhaupt Kulturanalyse? In: Franz Januschek (Hrsg.): Politische Sprachwissenschaft. Zur Analyse von Sprache als kulturelle Praxis. Opladen/Wiesbaden: Westdeutscher Verlag, S. 23-69.

Keppler, Angela (1994): Wirklicher als die Wirklichkeit? Das neue Realitätsprinzip in der Fernsehunterhaltung. Frankfurt a. M.: Fischer.

Klaus, Elisabeth/Margreth Lünenborg (2000): Der Wandel des Medienangebots als Herausforderung an die Journalismusforschung: Plädoyer für eine kulturorientierte Annäherung. In: $M e-$ dien E Kommunikationswissenschaft, 50(2), S. 188-211.

Klaus, Elisabeth/Margreth Lünenborg (2002): Journalismus: Fakten, die unterhalten - Fiktionen, die Wirklichkeit schaffen. In: Achim Baum/Siegfried Schmidt (Hrsg.): Fakten und Fiktionen. Konstanz: UVK, S. 152-164.

Klaus, Elisabeth/Stephanie Lücke (2003): Reality TV. Definition und Merkmale einer erfolgreichen Genrefamilie am Beispiel von Reality Soap und Docu Soap. In: Medien \& Kommunikationswissenschaft 51(2), S. 195-212.

Klaus, Elisabeth/Barbara O'Connor (2000): Pleasure and meaningful discourse. An overview of research issues. In: International journal of cultural studies, Heft 3, S. 369-387.

Klein, Hans (2003): Understanding WSIS: An Institutional Perspective on the UN World Summit on the Information Society. www.ip3.gatech.edu [9.12.2003]. 
Leder, Dietrich (2003): Wie bei Lothar Matthäus. Live vom Schlachtfeld: Die Kriegsberichterstattung wird sportifiziert. In: Berliner Zeitung vom 25.3.2003, S. 12.

Lindner, Rolf (1990): Die Entdeckung der Stadtkultur. Soziologie aus der Erfahrung der Reportage. Frankfurt a. M.: Suhrkamp.

Livingstone, Sonia (1999): Mediated knowledge: recognition of the familiar, discovery of the new. In: Jostein Gripsrud (Hrsg.): Television and Common Knowledge. London/New York: Routledge, S. 91-107.

Lünenborg, Margreth (2002): Journalismus kulturwissenschaftlich betrachtet. (Auch) Fiktionen liefern Ordnung. In: Michael Haller (Hrsg.): Die Kultur der Medien. Münster: Lit, S. 175188.

Lünenborg, Margreth (2004): Phänomene der Entgrenzung. Journalismus zwischen Fakt und Fiktion, Information und Unterhaltung. In: Udo Göttlich/ Mike Friedrichsen (Hrsg.): Diversifikation der Unterhaltungsproduktion. Köln: von Halem (im Druck).

Marshall, Thomas Humphrey (1992): Bürgerrechte und soziale Klassen: zur Soziologie des Wohlfahrtsstaates. Frankfurt a. M.: Campus.

Meyer, Thomas u. a. (2000): Die Inszenierung des Politischen. Zur Theatralität von Mediendiskursen. Wiesbaden: Westdeutscher Verlag.

Mikos, Lothar (2001): Fern-Sehen: Bausteine zu einer Rezeptionsästhetik des Fernsehens. Berlin: Vistas.

Mikos, Lothar/Feise, Patricia/Herzog, Katja/Prommer, Elisabeth (2000): Im Auge der Kamera. Das Fernsehereignis Big Brother. Berlin: Vistas.

Miller, Toby (1998): Technologies of Truth: Cultural Citizenship and the Popular Media. Minneapolis: University of Minnesota Press.

Moores, Shaun (1993): Interpreting Audiences. The Ethnography of Media Consumption. London/ Thousand Oaks/New Dehli: Sage.

Morley, David (1980): The Nationwide Audiences. Structure and Decoding. London: British Film Institute.

Morley, David (1992): Television Audience and Cultural Studies. London/New York: Routledge.

Morley, David (1999): Family Television. Cultural Power and Domestic Leisure. London/New York: Routledge.

Morley, David (2002): Television, Audiences and Cultural Studies. London/New York: Routledge.

Murdock, Graham (1994): Money talks: Broadcasting Finance and Public Culture. In: Stuart Hood (Hrsg.): Behind the screens. The structure of British Broadcasting in the 1990s. London: Lawrence \& Wishart, S. 155-183.

Murdock, Graham (1999): Rights and Representations: Public Discourse and Cultural Citizenship. In: Jostein Gripsrud (Hrsg.): Television and Common Knowledge. London/New York: Routledge, S. 7-17.

Ong, Aihwa (1996): Cultural Citizenship as Subject-Making. Immigrants negotiate racial and cultural boundaries in the United States. In: Current Anthropology, Heft 5, S. 737-762.

Ong, Aihwa (1999a): Flexible Citizenship. The cultural logics of transnationality. Durham/London: Duke.

Ong, Aihwa (1999b): Cultural Citizenship as Subject-Making: Immigrants Negotiate Racial and Cultural Boundaries in the United States. In: Rodolfo D. Torres, Louis F. Mirón, Jonathan Xavier Inda (Hrsg.): Race, Identity, and Citizenship. A Reader. Oxford/Malden: Blackwell, S. 262-293.

Rieger, Elmar (1992): Vorwort. In: Thomas Humphrey Marshall (Hrsg.): Bürgerrechte und soziale Klassen: zur Soziologie des Woblfahrtsstaates. Frankfurt a. M.: Campus, S. 7-32.

Rosaldo, Renato (1994): Cultural Citizenship in San José. California: Polar, S. 57-63.

Rosaldo, Renato (1999): Cultural Citizenship, Inequality and Multiculturalism. In: Rodolfo D. Torres/Louis F. Mirón/Jonathan Xavier Inda (Hrsg.): Race, Identity and Citizenship. A Reader. Oxford/Malden: Blackwell, S. 253-261.

Sarcinelli, Ulrich (1987): Symbolische Politik. Zur Bedeutung symbolischen Handelns in der Wahlkampfkommunikation der Bundesrepublik Deutschland. Opladen: Leske+Budrich. 
Storey, John (2003): Cultural Studies und die Populärkultur. In: Andreas Hepp/Carsten Winter (Hrsg.): Die Cultural Studies Kontroverse. Lüneburg: zu Klampen, S. 166-180.

Terkessidis, Mark (1999): Globale Kultur in Deutschland. In: Andreas Hepp, Rainer Winter (Hrsg.): Kultur - Medien - Macht. Cultural Studies und Medienanalyse. Opladen/ Wiesbaden: Westdeutscher Verlag, S. 237-252.

Thompson, Thomas (1990): Ideology and Modern Culture. Stanford CA.

Turner, Bryan S. (1994): Postmodern Culture/Modern Citizens. In: Bart van Steenbergen (Hrsg.): The Condition of Citizenship. London/Thousand Oaks/New Delhi: Sage, S. 153-168.

UNESCO (2003): Civil Society Representatives: No discrimination in providing access to information. http://portal.unesco.org/ci/ev.php?URL_ID=7653EURL_DO=DO_TOPICEURL_ SECTION=201 Ereload $=1044628904$ [11.2.2003].

UNESCO/ WSIS (2002): UNESCO and the World Summit on the Information Society. http://

portal.unesco.org/ci/ev.php?URL_ID=1543EURL_DO=DO_TOPICEURL_SECTION=201 Ereload=1051883714 [6.3.2003].

WACC (2002): Is the „Information Society“ a useful concept for Civil Society? www.wacc.org.uk/ publications/md/md2002-4/infosociety.html [17. 12. 2002].

WSIS (2002a): WSIS - World Civil Society Forum: Synthesis and Recommendations WG on Information Society. www.itu.int/wsis/documents [6.3.2003].

WSIS (2002b): WSIS - Subcommittee 2: World Summit Information Society. www.itu.int/wsis/documents [6.3.2003].

WSIS (2003a): WSIS - Civil Society Working Group on Content and Themes - Drafting Committee: Plan of Action: Civil Society's priorities. www.itu.int/wsis/documents [6.3.2003].

WSIS (2003b): Declaration of Principles. Dokument: WSIS-03/GENEVA/DOC/0004. www. itu.int/wsis/documents [12.12.2003].

WSIS (2003c): Plan of Action. Dokument: WSIS-03/GENEVA/DOC/0005. wwr.itu.int/wsis/documents [12.12.2003]. 